\title{
Convolutional Neural Network and Fuzzy Logic-based Hybrid Melanoma Diagnosis System
}

\author{
Fikret Yalcinkaya*, Ali Erbas \\ Department of Electrical and Electronics Engineering, Kirikkale University, \\ 71450 Yahsihan, Kirikkale, Turkey \\ fyalcinkaya@kku.edu.tr
}

\begin{abstract}
Studies on the detection of early stage melanoma have recently gained significant interest. Computer aided diagnosis systems based on neural networks, machine learning, convolutional neural networks (CNNs), and deep learning help early stage detection considerably. The colour and shapes of the images created by the pixels are crucial for the CNNs, as the pixels and associated pictures are interrelated just as a person's fingerprint is unique. By observing this relationship, the pixel values of each picture with its neighborhoods were determined by a fuzzy logic-based system and a unique fingerprint matrix named Fuzzy Correlation Map (FCov-Map) was produced. The fuzzy logic system has four inputs and one output. The advantage of CNNs trained with fuzzy covariance maps is to eliminate both the limited availability of medical grade training data and the need for extensive image preprocessing. The fuzzy logic output is fed to the pretrained AlexNet CNN algorithm. To deliver a reliable result, a deep CNN needs a large amount of data to process. However, to obtain and use the required sufficient data for diseases is not cost- and time-effective. Therefore, the suggested fuzzy logicbased fuzzy correlation map is tackling this issue to solve the limitedness of training CNN data set.
\end{abstract}

Index Terms-Fuzzy logic; Image segmentation; Medical diagnostic imaging; Neural networks.

\section{INTRODUCTION}

Cancer is a common word used to describe a range of related diseases. In its all forms and types, some associated cells begin to divide uncontrollably and penetrate into the surrounding tissues leading to cancer. In the case, older and damaged cells are not properly replaced, and then unexpected abnormal and uncontrollable extra cells, alien to the human body, lead to new structures called "tumours" or "cancer" [1]. Such a tumor type called "melanoma" starts in the skin and is the deadliest one during the last three decades. It develops when melanocytes, the cells that give the skin its tan or brown colour, start to grow out of control [2]. Melanoma spreads through metastasis, but medical statistics have shown that the survival rates depend highly on the stage of the cancer; so timely early stage detection creates higher chances of cure. The success rate of the dermatologists is less than $80 \%$ in effective skin cancer diagnosing, therefore new computerised or machine learning based and faster detection methods are needed. The three

Manuscript received 18 January, 2021; accepted 6 April, 2021. main conventional diagnosis techniques that proved positive for melanoma detection are ABCDE rule, 7 Point Checklist, and Menzies method [3]. Although melanoma prevalence is only about $1 \%$ of total skin cancers, malignant melanoma is the deadliest, especially in the developed countries, and the most rapidly increasing skin cancer type worldwide.

Diagnosis process goes through three steps: initial visual screening, dermoscopic analysis, and finally, a biopsy followed by histopathological examination that takes a lot of time, and by this time, the patient condition may greatly deteriorate. In another word, if a suspicious skin cancer is non-invasively verified by visual inspection, then histology is needed to make an explicit invasive diagnosis. It is usually operated on to remove the core melanoma region and a thin portion of normal skin surrounding the lesion. The removed part is then sent for biopsy analysis. If cancer is detected from the biopsy sample, then a second, but wider lesion removal may be carried out [4].

Dermoscopy is clinically being used to visualize the morphological structures in pigmented lesions due to two main parameters involved: uniform illumination and dense contrast. Although visual inspection proved positive in the non-invasive early diagnosis of melanoma, the limits of the human visual capacity lead to inaccuracies due to the morphological structures and their side effects. The inaccuracies might be due to many factors; among them, some are: hair presence, variable lesion sizes, colour and shape, irregular boundaries of asymmetrical lesions, or poor contrast regarding the normal skin and the lesion [5]. The interest in automatic image analysis methods is twofold: the relevance of quantitative information for a lesion and playing as an early warning tool. Digital image processing of melanoma lesions without biopsies is an effective way to detect skin cancer at an early stage. To reach this end, feature extraction is proved as an essential tool to analyse the image. Therefore, computerized analysis helps to extract features leading to early detection [6]. The major advantage of early diagnosis of melanoma is the decrease in melanoma mortality rates. Early dermoscopy detection of melanoma criteria is $\mathrm{ABCD}$ criteria, which must catch the attention when the size of the melanoma is relatively large in size; diameter (D) $>6 \mathrm{~mm}$ [7]. At this stage, minor surgery enhances the chances of patient recovery if the lesion is in its early stages [8]. Automated lesion segmentation is an 
essential target in computer-aided melanoma diagnosis approaches. The computer-aided diagnosis methods use the parameters for feature extraction, such as significant variations detected in location, shape, size, colour, and texture of the lesions. The feature extraction and classification steps of the lesions are directly affecting the accuracy of the segmentation process due to border irregularity and lesion asymmetry. Feature based extraction of lesions from medical grade images use three methods. These techniques are to expose the lesions to a threshold value, to use the method of active contours, and finally, to merge regions of interest accordingly [9]. Although these features usually use pixel-level information, the representation of high-level attributes or semantic information, in complex dermoscopic images and their signal processing, these conventional methods are not satisfactory enough and they fail to provide enough clinically relevant results [10]. In fully automated segmentation methods, correct and accurate examination of every single pixel's image requires experts' decision based determination of the region of interest and its extremely sensitive and thinner boundary with the nearest neighborhood. The source of some additional difficulties arising due to the automated segmentation of medical images is the wide variations in the shape, size, and texture of the images, and in some specific cases, the colour of the region of interest and poor contrast between regions [11]. Advanced treatment needs new integrative and efficient methods leading to possible early detection of melanoma via image processing. Many challenges arise due to the difficulty of indistinguishable boundaries of the tumor with the healthy natural skin. Physical and various conditions of specific cases of lesions must be processed in such a way that targeted early detection and diagnosis of melanoma must be realized [12]. The superiority of convolutional neural networks (CNNs), including the analysis of dermoscopic images, is due to its ability to learn automatically the hierarchical features from raw data provided as input and reflecting back higher levels of representation, and being more robust than manually controlled clinical conventional practice [13]. Furthermore, CNN architecture, using deep learning, has achieved its success via the use of three main tools: segmentation, object detection, and classification. This success is primarily attributed to the capability of high-level of semantic meaning extraction from image feature models [14]. Deep neural networks (DNNs) learn high-level features, e.g., edges, from low-level features, e.g., pixel intensities [15]. DNN architecture uses multilayer CNN in comparison with traditional artificial neural networks. The use of the deep network architecture gives the opportunity to extract automatic features without human interference. The extracted normal and complex features are the natural result of the number of hidden layers used. New melanoma detection methods are also needed; therefore, a hybrid architecture combining different techniques to recognise and segment the dermoscopic images is available [16].

\section{THEORY AND METHODS}

Skin lesion classification using deep-learning approach, particularly targeting early melanoma detection, was used to detect and classify a dermoscopic image containing a skin lesion. An AlexNet based CNN architecture integrated with the transfer learning paradigm was proposed for skin lesion classification effectiveness in terms of time and cost [17]. Targeting the classification of skin cancer images as benign or malignant, an application based on $\mathrm{CNN}$ via AlexNet was developed. This CNN based application permits easy access for medical diagnosis and is targeted for the use of underdeveloped countries [18]. Any novel method based on deep learning for the extraction of lesion region requires preprocessing to reduce artefacts; the input image is then given to the deep $\mathrm{CNN}$, where it produces a segmentation mask of the lesion region [19]. A deep CNN based learning method was proposed for segmentation of skin lesions of clinical images after proper preprocessing; however, CNN is a usable tool with relatively less preprocessing algorithms as well [20]. To evaluate skin lesions from dermoscopic images using CNNs via transfer learning method has also been tested [21]. Melanoma recognition uses two established methods to decide either very deep CNNs or low-level features or substantially deeper networks with more than 50 layers. Very deep CNNs have the ability and capacity to fetch rich texture and more discriminative features for detection and recognition [22]. A system integrating deep learning with machine learning approaches based on recent research has also been developed, creating a new type of methods interconnection capable of segmenting skin lesions properly [23]. Accurate skin lesion segmentation is a core issue in the computer-aided diagnosis of melanoma. However, some performance of segmentation methods is weak. The reasons are the lesions' fuzzy boundaries, uniform illumination, and low contrast of the image, background effects, inhomogeneous textures, or artifacts.

A computer vision application needs high-quality medical grade images. However, images captured under weak illumination conditions are subjected to degradation of crucial parameters, such as degradation of visibility, brightness, and contrast. Weakly illuminated image needs preprocessing to enhance the image. Due to the role and importance of degraded images, various methods have been developed. The issues that need interest regarding the weak illumination problems of the images are two folds: quantitative and qualitative image enhancement. Quantitative and qualitative images need accurate and varying illumination conditions, not degraded nature of the image (lesion), and effective details of the image texture. However, not to be forgotten is the penetrating effect of changing illumination conditions, such as non-uniformity of illumination, its back lighting environment, illumination intensity, and well-lit conditions [24].

Research focuses on a system capable of assessing the seriousness of melanoma using dermatological grade photographs of the skin lesion. However, photographs of the lesion have different grades of illumination, which adversely affect the lesion segmentation and classification performance. The primitive forms of automated systems used dermoscopy images to assess the degree of risk of melanoma. However, there are some other sources used to take images, such as the digital dermascope images. It is a device that magnifies the surface of the lesion in detail and 
filter the surface reflectance as well. Nevertheless, this instrument is not widely used by medical experts due to various reasons. A more widely used method by dermatologists is the images taken by a digital camera. Then the lesion areas are determined by properly segmenting the pictures of the images, and then the features are extracted. Finally, the lesion is assessed and classified to determine the risk level of the lesion, whether it is melanoma or not. The segmentation, extraction, and grade assessment are all affected by the illumination from skin surface reflectance. Due to that, healthy skin areas covered with dense shadows lead to misclassification of those areas [25].

Additionally, the performance of the methods depends on various parameters and on the use of preprocessing techniques. The lesion segmentation and boundary extraction in images are often ill-defined due to the transition from the lesions to the surrounding healthy skin or vice versa. Indeed, most skin lesions have fuzzy boundary transitions from the highly dense (core region) to the surrounding light natural tissues. Although various regionbased segmentation methods can be used for skin lesion detection, colour and gray scale thresholding is the most commonly used technique. Therefore, these methods are mostly not capable enough to define the required types of criteria to help precisely separate the pigmented lesions from the surrounding healthy skin safely [26].

CNN algorithms are very successful against conventional image processing, such as threshold or edge based. Despite this success, there are some factors that limit the success of CNN algorithms. The most important of these factors are: the need to be trained with a lot of data and their sensitivity to external factors, such as brightness and colour temperature of the light source.

- Medical image processing requires anonymized, highquality, structured, and labelled data. Deep learning and Deep CNN need a large amount of data to deliver reliable results. However, the fetching and usage of the data, concerning the seriousness of medical problems, are unfortunately not cost- and time-effective. Fortunately, there are some techniques tackling this issue. One is data augmentation, which helps to overcome the overfitting and improves the accuracy. Various augmentation methods are applicable to medical data, such as shifting, zooming, flipping, and rotating. These widely preferred methods used to overcome the data augmentation problem are known as training time data augmentation methods. Transfer learning is another performance enhancing method in deep models, especially in CNNs [27]. In conventional CNN applications, the image with the lesion is given as input to the CNN network and the network architecture designed uses the colour information provided by every single pixel available. In the case of network applications having limited data, the data provided by the colour information of every single pixel available are not enough for proper image processing. Therefore, it is needed to provide extra healthy data already present in the picture used as input to the CNN network. The source of this extra healthy data is pixels and their interconnected relationship with the nearest neighborhood, such as the relation of the pixel forming the original image with other pixels, the quantity or frequency of the crucial pixel forming the lesion part, the pixel's distance to the center of the image, the surface texture created as a result of the interconnection of pixels; all of those parameters provide crucial information regarding detection of the lesion region (or region of interest). Shortly, it is possible to fetch enough and crucial information from images, which are highly dense, but less in quantity than from images which are higher in quantity, but weaker carriers of information.

- Second factor needing attention is the structure of the input image, as it consists of two parts, a healthy part (normal skin) and an unhealthy part (the lesion). This binary structure may mislead the training of $\mathrm{CNN}$ algorithm because the texture of the healthy areas provides irrelevant parameter(s) for melanoma detection. At the same time, the outturn of the healthy areas could cause the loss of relevant information regarding the lesion. To reduce the negative effects of the normal skin's texture on the classification process, the CNN needs extra image processing steps, such as segmentation mask, or colour plane transformation [28]. This paper is proposing a new method for reducing the effects of the normal skin's texture on the classification process. The morphological structure of skin texture is based on pixels colour differences; therefore, the pixels relationship with its close environment may transfer a great deal of unsafe data from pixels to CNN architecture.

Correlation statistics can be used to create images. In spatial data analysis, correlograms are images obtained by correlation statistical techniques. The spatial data analysis methods produce results using the location of the object under scrutiny. Colour correlogram vectors show the colour correlation of neighborhood pixels in the indexed images.

In this paper, a user-defined vector named as "Fuzzy Correlation Map" is presented. It discloses the correlation of neighborhood pixels with the active pixel and the same algorithm applies to the whole image. On the contrary, the colour correlograms calculate colour distribution of the lesion. In other words, the development of the Fuzzy Correlation Map (FCM) is an improved version of the colour correlogram method (CCM). The colour correlogram method derives a feature vector of each image database by computation and stores and saves them as their colour image. Whereas FCM computes a feature vector of each image database and stores the associated values as their image data values. In both systems, CCM and FCM, the vectors of tested images are first calculated and then compared both with the trained images and the feature vectors of the trained images, respectively. As a result, the Fuzzy Correlation Map with its original algorithm reduces computational cost. To effectively reduce the computational cost, correlograms using small distance values have been used [29].

The ambient light effect in the pictures of the training set used here affects the lesion area, as well as every part of the picture, including the borders of the lesion or areas without lesions. Therefore, it can be said that although the relationships of the pixels forming the picture are affected independently from ambient light and formation, such as brightness, their relations with each other are not affected.

Developed fuzzy logic-based Fuzzy Correlation Map is 
used to overcome not only the uniform illumination, but also misleading ambient light problems by a limited number of lesion images, which is used in training CNN data problem.

A new method has been proposed to remove the problems mentioned here. Limited training data are obtained from 30 lesion images randomly selected from the ISIC database. The developed method has been trained and tested with 120 images obtained by image augmentation, such as flipping on $\mathrm{x}$ axis, $\mathrm{y}$ axis, and rotating 180 degrees. The developed method enabled 120 images to be used without applying image preprocessing, such as brightness and histogram equalization.

Deep CNNs based image segmentation processing methods eliminate all sorts of secondary affects, especially in clinical images via a method integrating pretrained deep learning network and transfer learning method. In addition to the widely used two methods, fine-tuning and data augmentation, the method of transfer learning is also applied to AlexNet by replacing the last layer by a softmax, being able to classify melanoma or non-melanoma (benign) cases. The modified AlexNet architecture is given in Table I.

TABLE I. THE MODIFIED ALEXNET ARCHITECTURE [30]

\begin{tabular}{|c|c|c|c|c|c|}
\hline Layer & Input Dimension & Stride & Kernel Dimension & Padding & Output Class \\
\hline Convolution & $227 \times 227 \times 3$ & 4 & $11 \times 11$ & 0 & 96 \\
\hline Maxpooling & $55 \times 55 \times 96$ & 2 & $3 \times 3$ & 0 & 96 \\
\hline Convolution & $27 \times 27 \times 96$ & 1 & $5 \times 5$ & 3 & 256 \\
\hline Maxpooling & $55 \times 55 \times 96$ & 2 & $3 \times 3$ & 2 & 256 \\
\hline Convolution & $27 \times 27 \times 96$ & 1 & $3 \times 3$ & 2 & 384 \\
\hline Convolution & $27 \times 27 \times 96$ & 1 & $3 \times 3$ & $3 \times 34$ & 256 \\
\hline Convolution & $27 \times 27 \times 96$ & 1 & $3 \times 3$ & & 256 \\
\hline Maxpooling & $55 \times 55 \times 96$ & 2 & $1 \times 1$ & & 4096 \\
\hline Fully Conn. & & & $1 \times 1$ & & \\
\hline Fully Conn. & & & $1 \times 1$ & & 2 \\
\hline Fully Conn. & & & & & 2096 \\
\hline
\end{tabular}

The automated melanoma detection architecture used in this paper is based on a deep CNN learning method combined with fuzzy logic-based method to produce the fuzzy correlation map of the pixels' image fed to CNN network. Tested on a publicly available large ISIC dataset, the model demonstrated high classification accuracy, sensitivity, and specificity when compared with non-fuzzy correlation map images fed to classifiers tested on the same dataset [31].

Just as each picture is unique in itself, this paper showed that the correlation between the pixels creating the picture is unique in itself as well. The relationship between the pixels forming the image picture is determined by a fuzzy logicbased architecture, and the acquired data is fed to the CNN network, so as a new dimension has been added to help a better the level of the diagnosis of melanoma. Suggested architecture components are processed via appropriate filters and activation maps are created, and the information is resized by pooling operations, and finally, transferred to the last part of the total network, the artificial neural network. Then the image file with lesion images is fed to the CNN system and the system produces an output of the lesion in the image. In many applications of CNN, the input data provided are not sufficient to train a new deep CNN architecture. To overcome this issue, the theory of transfer learning is used to pretrained $\mathrm{CNN}$ based application. Using pretrained $\mathrm{CNN}$ network in critical applications, it is expected to increase the accuracy. The advantage of the developed fuzzy logic-based image enhancement system is its ability to improve $\mathrm{CNN}$ detection capacity sufficient enough by training AlexNet with a small number of dermoscopic images [32].

\section{A. Fuzzy Correlation Map (FCore-Map)}

The Fuzzy Correlation Map (FCore-Map) is a $227 \times 227$ dimension matrix, whose elements have pixel energy calculated via fuzzy logic system with covariant matrix, distance vector matrix, image matrix, and compass operator matrix. The original lesion image is shown in Fig. 1 below, from which four input matrixes of the fuzzy logic system are calculated.

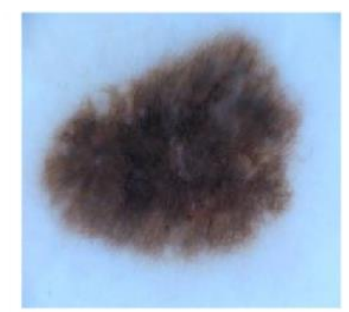

Fig. 1. Original image.

\section{Covariance Map.}

The covariance is a variance measuring of how two variables change with respect to each other. Covariance matrix-based analysis has been widely used in various areas of research, such as in pattern recognition-based applications, computer vision, and signal processing. Covariance encodes the distribution information within the data. It is used to model the data dependency irrespective of physical meaning. For example, the covariance can be used to represent the dependency between heterogeneous statistical data, such as pixel intensity, position, and their relation with derivatives. This inspired researchers that it might be possible to use the covariance matrix to integrate data gathered into deep learning frameworks [33]. If two randomly variable vectors are present, $A$ and $B$, then their covariance is defined as $\operatorname{cov}(A, B)$, where $\mu_{A}$ is the mean of $A, \mu_{B}$ is the mean of $B$, and $*$ denotes the complex conjugate. To find the covariance matrix value of two randomly changing vectorial data, it is needed to find a new matrix. A pairwise covariance calculation between each variable given by (1) is [34] 


$$
\operatorname{cov}(A, B)=\frac{1}{N-1} \sum_{i=1}^{N}\left(A_{i}-\mu_{A}\right) *\left(B_{i}-\mu_{B}\right)
$$

If $A$ is a matrix with random variables obtained due to the measurement recorded of the image lesion, then the covariance matrix can be obtained by the pairwise covariance calculation between each column combination given as follows (2) [34]

$$
C=\left(\begin{array}{ll}
\operatorname{cov}(A, A) & \operatorname{cov}(A, B) \\
\operatorname{cov}(B, A) & \operatorname{cov}(B, B)
\end{array}\right)
$$

The definable matrix elements of matrix $C(i, j)$ are equal to $\operatorname{cov}(A(:, i), A(:, j)$, where $\mu$ is the mean of $A$ given as follows on (3) [34]

$$
\mu=\frac{1}{N} \sum_{i=1}^{N} A_{i}
$$

The covariance matrix model output is given in Fig. 2.

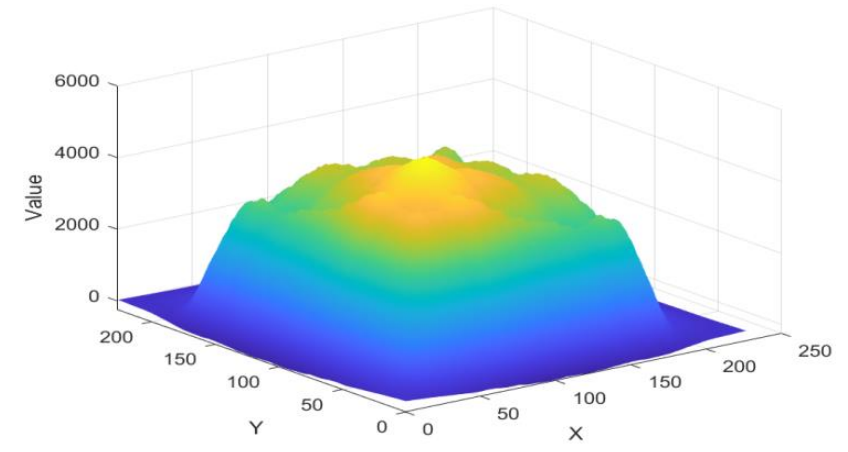

Fig. 2. The covariance matrix.

\section{Compass Mean Operator.}

The compass operator acts like a compass on top of a map, so as the compass operator signs or directs the neighborhood pixels properly and calculates correlation between the central pixel and neighbouring pixels to obtain the mean intensities via fuzzy logic. The main difference between the average mean filters is the center pixel not being used. The $\Delta p$ function is defined as follows, with zero padding applied on all sides given on (4) is

$$
\Delta p(i, j)=\frac{1}{m \times n} \sum_{\substack{-1 \leq m \leq 1 \\-1 \leq n \leq 1}} P(i, j)
$$

where $1 \leq i \leq 227$ and $1 \leq j \leq 227, i \neq j$.

Compass Mean Operator output is given in Fig. 3.

The filtering method is a widely used method to average the neighborhoods of the pixels that make up the picture. The effect of contradictory pixels on the surface texture can be reduced and more accurate training can be provided thanks to the homogeneous structure obtained. Furthermore, the image becomes clear and unwanted artifacts, such as hair, are removed. The reason the central pixel is not used is that the image itself is included in another fuzzy logic login membership function, so the relevant information comes from the other login membership function.

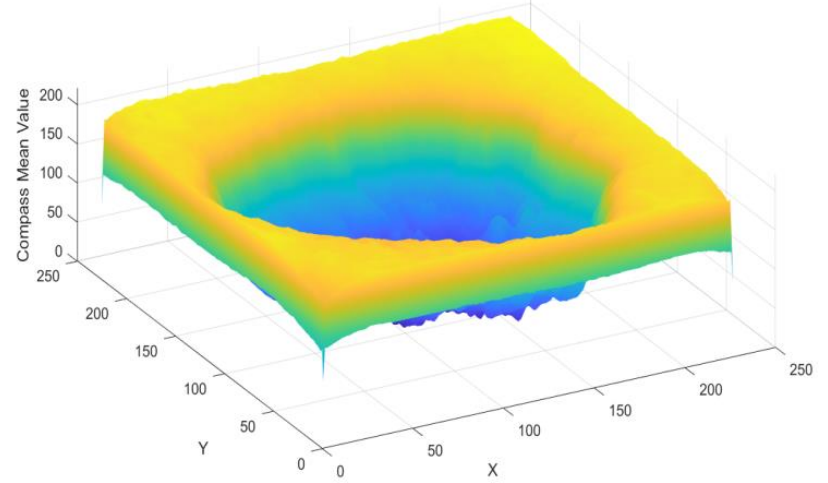

Fig. 3. Compass Mean Operator output.

\section{Distance Vector Matrix.}

Distance vector is a scalar quantity measuring the distance between pixels position and the center of the image given by (5) below

$$
D p(i, j)=\sqrt{\left(i-c_{x}\right)^{2}+\left(j-c_{y}\right)^{2}} .
$$

The coordinates $c_{x}$ and $c_{y}$ are the active center of the image, where each $227 \times 227$ pixel image is defined as $\left(c_{x}, c_{y}\right)$ $=(113,113)$, respectively. The distance vector matrix of the image and the values of the elements of the matrix are graphically shown in Fig. 4.

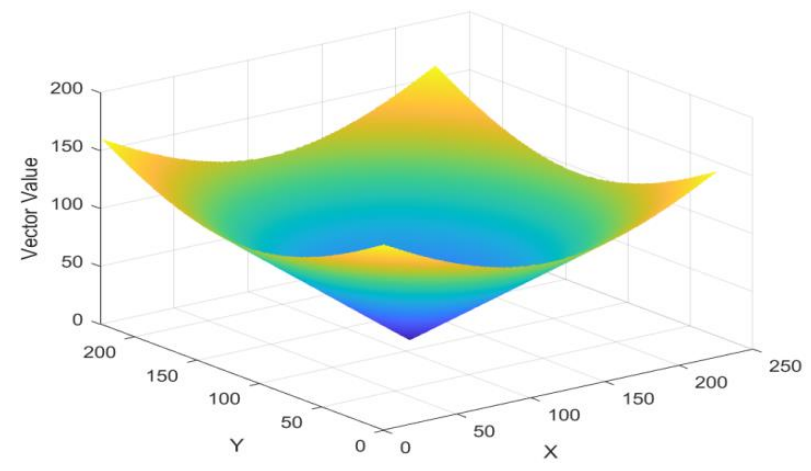

Fig. 4. Distance vector matrix.

When the dermoscopic pictures were examined, it was seen that the lesion area was in the center of the picture. Therefore, it is seen that each pixel carries meaningful information about its distance from the center.

4. Grayscale Image Matrix (Pixel Intensity Matrix).

Lesion image on the dataset is converted to a grayscale image and saved as a matrix to input the fuzzy logic model. The elements of the grayscale image matrix define intensity values from 0 to 255 . Although the information derived from the pixels that make up the image is used, the original information of the image is also used. Grayscale image of the lesion is given in Fig. 5.

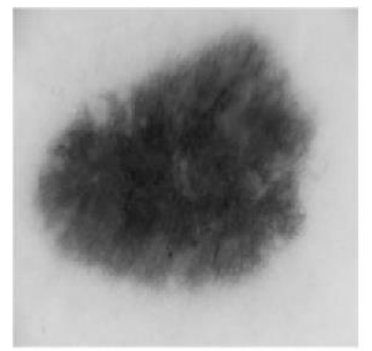

Fig. 5. Grayscale image of lesion. 


\section{B. Fuzzy Logic-Based Fuzzy Correlation Map (FCore- Map) Calculation}

The fuzzy logic system has four input membership functions and one output function. The input functions are compass operator matrix, pixel intensity matrix, distance vector matrix, and covariance matrix, respectively. The output is the correlation energy of pixels' light intensity taking values in the range from 0 to 255 . The output matrix is a gray-scale image matrix holding the correlation between total pixels' intensity energy.

The designed fuzzy logic system is given in Fig. 6.

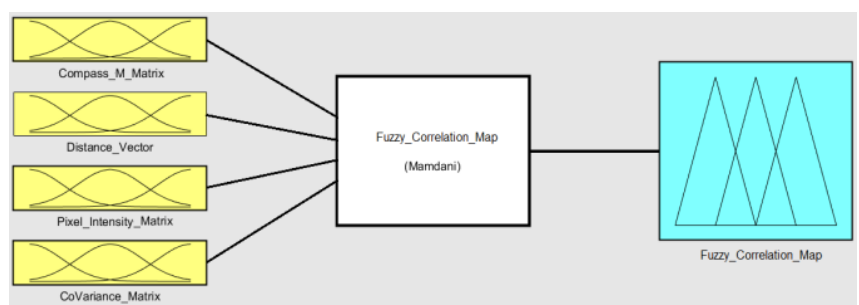

Fig. 6. The designed fuzzy logic system.

The four inputs and one output membership functions are given in Fig. 7(a), Fig. 7(b), Fig. 7(c), Fig. 7(d), and Fig. 7(e), respectively.
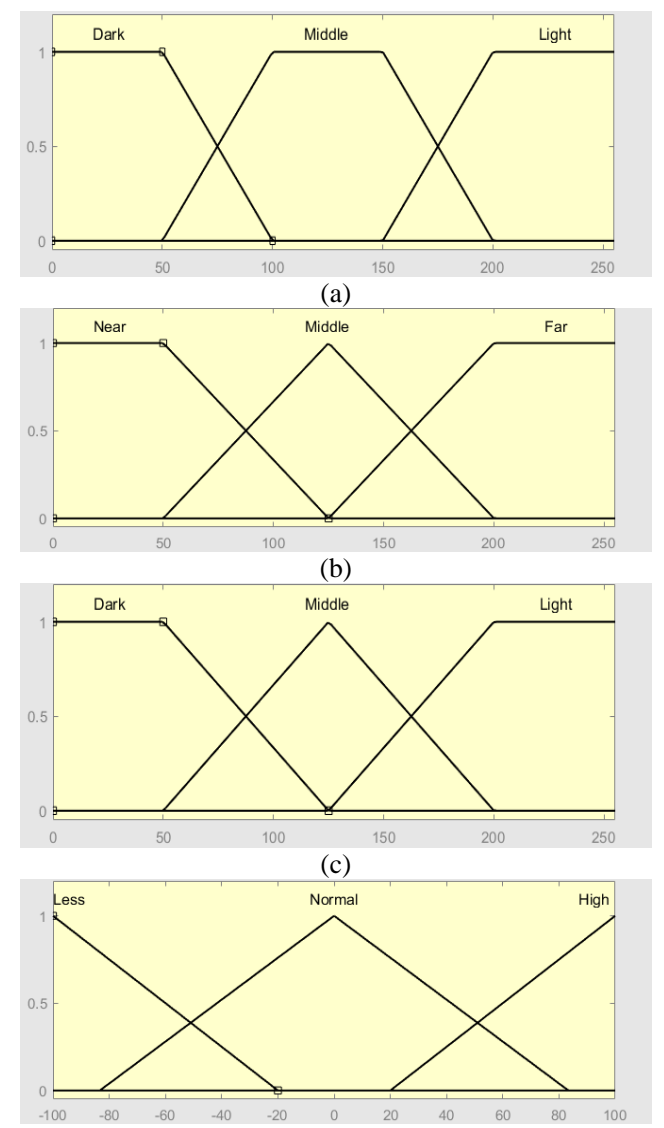

(d)

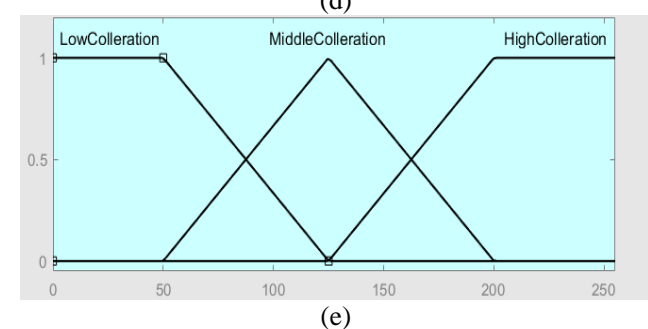

Fig. 7. Membership functions of inputs and output: a) Compass_Mean_Matrix; b) Distance_Vector; c) Pixel_Intensity_Matrix; d) CoVariance_Matrix; e) Fuzzy_Correlation_Map.
The designed fuzzy logic system has four membership functions for input and only one membership function for the output. A graphical representation of the fuzzy variable sets simply produces a proper membership function. For example, the distance_vector membership function has three fuzzy sets: near, middle, and far. The rule base defined directs the definition rules for membership functions, and the created rules determine the membership function behavior of the total system. Eighty-one rules are determined for the system developed. One of the rules is given below:

- If Compass_Mean_Matrix is Dark and Distance_Vector

is Near and Pixel_Intensity_Matrix is Dark and CoVariance_Matrix is Less, then the Output is Low_Correlation.

In the proposed fuzzy logic-based $\mathrm{CNN}$ image feed system, the rule base system of the input membership functions is extended to the output membership function to produce the required output. The inputs are compass mean matrix, distance vector, pixel intensity matrix, and covariance matrix. The output is Mamdani based generated Fuzzy Correlation Map.

The fuzzy correlation map produced by fuzzy logic system is given by the image shown in Fig. 8 .

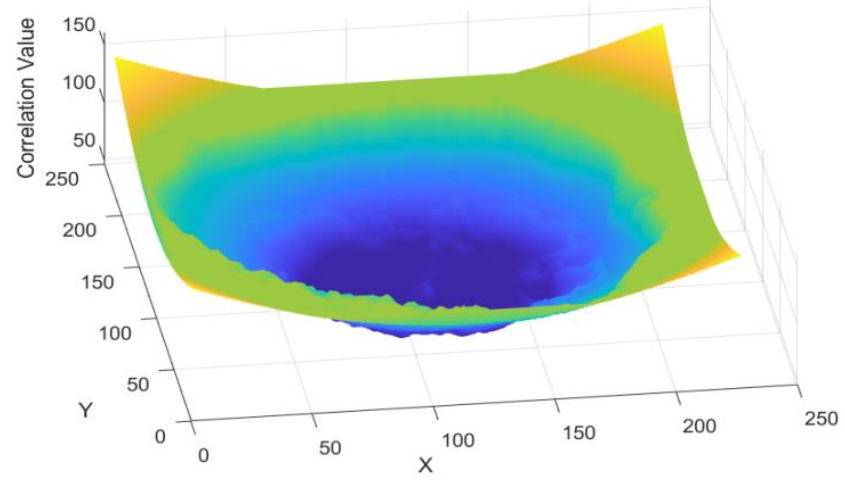

Fig. 8. The fuzzy correlation map produced by fuzzy logic system.

\section{The Developed CNN Based System}

This paper uses the CNN architecture, which has two stages for the diagnosis application of melanoma. The first stage accepts conventional dermatological images as inputs to fuzzy logic system. Fuzzy logic system enhances the images before inputting to CNN network. The total system finally decides whether the image is a melanoma or a benign lesion. In the proposed CNN network, the theory of transfer learning is applied to the pretrained and modified AlexNet architecture. The classification layer is modified with two classes for this application. The basic blocks of the architecture main layers, pretrained and modified AlexNet with fuzzy logic, are detailed as follows and given in Table I [20].

\section{- Convolutional Layer.}

CNN architecture has hidden layers as well, such as convolutional layers, pooling layers, fully connected layers, and normalization layers. The architecture of the convolutional layer uses a certain number of filters applicable to the input image, based on the requirements and size of the input image; the first layer of the developed architecture has a filter with $11 \times 11$ dimensional structure. The input image size is $224 \times 224 \times 3$, but due to padding, it 
turns out to be $227 \times 227 \times 3$. Each filter and the input image are independently convolved. Therefore, the result of each convolution operation is a $2 \mathrm{D}$ image map, the feature map. Convolutions by filters enable each filter to recognize and differentiate between different and distinctive features therein.

\section{- Rectified Linear Unit Layer (ReLU).}

ReLU is expected to increase the nonlinearity of the network in such a way that the comprehensive area of each layer must stay unchanged as a result of being a nonsaturated function. ReLU based network training is significantly faster than other activation functions, such as tanh and sigmoid.

- Max-pooling Layer.

The pooling layer reduces the size of the input image by downsampling. The input image is divided into nonoverlapping rectangular matrices to attend preprocessing, then max-pooling returns the maximum value of each matrix representing the lesion region. Max-pooling makes dimensional reduction possible on the data processed.

- Fully Connected Layer.

The function of the fully connected layer in CNN architecture is to connect all neurons originating from and contributed to the previous layers. The $2 \mathrm{D}$ outputs, the images from the previous layers, are reshaped to the 1D output layer before the classification process is started.

The basic architecture of the designed Fuzzy logic and CNN based diagram is given in Fig. 9. The developed CNN based system architecture has cascaded built-in multilayer two-level structure and functions. The first step of the architecture produces fuzzy logic-based fuzzy correlation map via pixels' and creative textures of the created images, and in the second step the created map is given as an input to the modified AlexNet architecture.

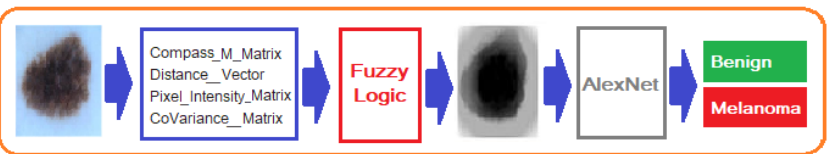

Fig. 9. The basic architecture of the designed fuzzy logic system and the modified AlexNet based CNN diagram.

In conventional applications, the images of the lesion area are given to AlexNet as inputs, whereas the system we developed uses the output of the fuzzy correlation map as inputs to the AlexNet. This is the main difference and the original contribution of the system suggested, and this attribute is unique in itself, differentiating the proposed architecture from the rest in the literature. As the colour information of each single image is its fingerprint, so as it is possible to define the fuzzy correlation map, which carries deep, undetected and more information of the image, as the finger prints of the image as well. The developed Matlab based fuzzy logic algorithm accepts four basic membership functions as inputs and outputs only one function, the fuzzy correlation map. The produced map, named as the "fuzzy correlation map", is used to train and test the AlexNet algorithm. Having completed the network training successfully, then it is needed to decide the region of interest. To do that, the fuzzy correlation map of the lesion image area is obtained, and then the available Fuzzy
Correlation Map is applied to the trained AlexNet, which is expected to predict the lesion area (area of interest).

\section{DISCUSSION}

In this paper, a new method named as "Fuzzy Correlation Map" using fuzzy logic to feed CNN architecture to enhance limited dataset performance was developed. The integrated new fuzzy logic and CNN based model was performed on ISIC image dataset. The quantitative evaluation of the method is based on the evaluation and calculation of three parameters: sensitivity, specificity, and accuracy. The function of the segmentation process is to classify each pixel into two classes of lesions, either positive (melanoma) or negative (non-melanoma). As a result, to compare the output of the developed fuzzy logic-based $\mathrm{CNN}$ architecture with one of the well-established references, the AlexNet architecture, then the classification metrics are required to be calculated accordingly, using the definitions given in the equalities given below [19]:

$$
\begin{gathered}
\text { Sensitivity }=T P /(T P+F N), \\
\text { Specificity }=T N /(T N+F P), \\
\text { Accuracy }=\frac{T P+T N}{T P+F N+T N+F P} .
\end{gathered}
$$

The confusion matrix of the diagnosis system is given in Table II.

TABLE II. CONFUSION MATRIX.

\begin{tabular}{|c|c|c|c|}
\hline \multicolumn{2}{|c|}{} & \multicolumn{2}{|c|}{ Actual } \\
\cline { 3 - 4 } \multicolumn{2}{|c|}{} & Positive & Negative \\
\hline \multirow{2}{*}{ Predicted } & Positive & TP (True Positive) & FP (False Positive) \\
\cline { 2 - 4 } & Negative & FN (False Negative) & TN (True Negative) \\
\hline
\end{tabular}

The performance of the proposed method is compared with ordinary lesion images on ISIC image database fed to AlexNet, and the results are compared as in Table III with their best quantitative values obtained. The proposed method attains better performance in terms of sensitivity, specificity, and accuracy in comparison with AlexNet outputs both for using normal input images and improved image maps.

TABLE III. THE COMPARISON OF THE SYSTEM SUGGESTED WITH THE ALEXNET.

\begin{tabular}{|c|c|c|c|}
\hline & Sensitivity & Specificity & Accuracy \\
\hline AlexNet & 0.50 & 0.78 & 0.77 \\
\hline $\begin{array}{c}\text { AlexNet with fuzzy logic } \\
\text { (developed system) }\end{array}$ & 0.54 & 0.82 & 0.80 \\
\hline
\end{tabular}

Generally, different applications either use images (lesions) with image augmentation or without image augmentation applied. However, the contribution of this paper is concisely based on the four crucial inputs. Those inputs are named as "CompassMeanMatrix", "DistanceVector", "PixelIntensityMatrix", and most importantly "CoVarianceMatrix". In the system designed, these four input variables are obtained from the input image (lesion) and then fed to fuzzy logic, where the parameters go through fuzzy logic-based processing leading to the 
Correlation vector map, and finally, this matrix is fed as an input to the AlexNet, where it decides about whether the lesion is melanoma or not.

\section{CONCLUSIONS}

Conventional methods, including image-processing techniques, are required, but not sufficient enough to detect melanoma as early as possible. Due to the developments in machine learning, $\mathrm{CNN}$, and deep learning, innovative new methods have been developed leading to their integration with conventional methods already present. To create an innovative method, this paper combined the AlexNet with Fuzzy Logic. The three-parameter values obtained as a result of the integrated form of AlexNet with Fuzzy Logic are sensitivity (0.54), specificity (0.82), and accuracy (0.80) when compared with AlexNet. These results were obtained via Fuzzy Correlation Map, given as an input to the AlexNet system. The designed integrated fuzzy logic system operates on the melanoma images to eliminate the high-quality medical image requirement for network training. The system developed is capable of extracting the hidden data in the pixels and their neighbouring environment.

The colour correlogram method derives a feature vector of each image database by computation and stores and saves them as their colour images. Whereas FCM computes a feature vector of each image database and stores the associated values as their image data values. In both systems, CCM and FCM, the vectors of tested images are first calculated and then compared both with the trained images and the feature vectors of trained images, respectively. As a result, the Fuzzy Correlation Map with its original algorithm reduces the computational cost. To effectively reduce the computational cost, correlograms using small distance values were used.

\section{CONFLICTS OF INTEREST}

The authors declare that they have no conflicts of interest.

\section{REFERENCES}

[1] “What Is Cancer?", National Cancer Institute. [Online]. Available: https://www.cancer.gov/about-cancer/understanding/what-is-cancer

[2] "What Is Melanoma?", American Cancer Society. [Online]. Available: https://www.cancer.org/cancer/melanoma-skincancer/about/what-is-melanoma.html

[3] V. Pomponiu, H. Nejati, and N.-M. Cheung, "Deepmole: Deep neural networks for skin mole lesion classification", in Proc. of 2016 IEEE International Conference on Image Processing (ICIP), 2016, pp 2623-2627. DOI: 10.1109/ICIP.2016.7532834.

[4] M. A. Kadampur and S. A. Riyaee, "Skin cancer detection: Applying a deep learning based model driven architecture in the cloud for classifying dermal cell images", Informatics in Medicine Unlocked, vol. 18, article 100282, 2020. DOI: 10.1016/J.IMU.2019.100282.

[5] R. Mishra and O. Daescu, "Deep learning for skin lesion segmentation", in Proc. of 2017 IEEE International Conference on Bioinformatics and Biomedicine (BIBM), 2017, pp. 1189-1194. DOI: 10.1109/BIBM.2017.8217826.

[6] M. E. Celebi, G. Schaefer, H. Iyatomi, and W. V. Stoecker, "Lesion border detection in dermoscopy images", Comput. Med. Imaging Graph., vol. 33, no. 2, pp. 148-153, 2009. DOI: 10.1016/J.COMPMEDIMAG.2008.11.002.

[7] G. Argenziano, G. Albertini, F. Castagnetti, B. De Pace, V. Di Lernia, C. Longo, G. Pellacani, S. Piana, C. Ricci, and I. Zalaudek, "Early diagnosis of melanoma: What is the impact of dermoscopy?", Dermatol. Therapy, vol. 25, no. 5, pp. 403-409, 2012. DOI: 10.1111/j.1529-8019.2012.01482.x.

[8] A. Naeem, M. S. Farooq, A. Khelifi, and A. Abid, "Malignant melanoma classification using deep learning: Datasets, performance measurements, challenges and opportunities", IEEE Access, vol. 8, pp. 110575-110597, 2020. DOI: 10.1109/ACCESS.2020.3001507.

[9] Y. Yuan and Y.-C. Lo, "Improving dermoscopic image segmentation with enhanced convolutional-deconvolutional networks", IEEE Journal of Biomedical and Health Informatics, vol. 23, no. 2, pp. 519-526, 2019. DOI: 10.1109/JBHI.2017.2787487.

[10] F. Xie, J. Yang, J. Liu, Z. Jiang, Y. Zheng, and Y. Wang, "Skin lesion segmentation using high-resolution convolutional neural network", Comput. Methods Programs Biomed., vol. 186, article 105241, 2020. DOI: 10.1016/J.CMPB.2019.105241.

[11] I. R. I. Haque and J. Neubert, "Deep learning approaches to biomedical image segmentation", Informatics in Medicine Unlocked, vol. 18, article 100297, 2020. DOI: 10.1016/J.IMU.2020.100297.

[12] T. Y. Tan, L. Zhang, and C. P. Lim, "Adaptive melanoma diagnosis using evolving clustering, ensemble and deep neural networks", Knowledge-Based Systems, vol. 187, article 104807, 2020. DOI 10.1016/J.KNOSYS.2019.06.015.

[13] F. Xie, J. Yang, J. Liu, Z. Jiang, Y. Zheng, and Y. Wang, "Skin lesion segmentation using high-resolution convolutional neural network", Computer Methods and Programs in Biomedicine, vol. 186, article 105241, 2020. DOI: 10.1016/J.CMPB.2019.105241.

[14] L. Bi, J. Kim, E. Ahn, A. Kumar, M. Fulham, and D. Feng, "Dermoscopic image segmentation via multistage fully convolutional networks", IEEE Trans. Biomed. Eng., vol. 64, no. 9, pp. 2065-2074, 2017. DOI: 10.1109/TBME.2017.2712771.

[15] S. Sabbaghi, M. Aldeen, and R. Garnavi, "A deep bag-of-features model for the classification of melanomas in dermoscopy images", in Proc. of 2016 38th Annual International Conference of the IEEE Engineering in Medicine and Biology Society (EMBC), 2016, pp. 1369-1372. DOI: 10.1109/EMBC.2016.7590962.

[16] M. P. Pour, H. Seker, and L. Shao, "Automated lesion segmentation and dermoscopic feature segmentation for skin cancer analysis", in Proc. of 39th Annual International Conference of the IEEE Engineering in Medicine and Biology Society (EMBC), 2017, pp. 640-643. DOI: 10.1109/EMBC.2017.8036906.

[17] A. R. Lopez, X. Giro-i-Nieto, J. Burdick, and O. Marques, "Skin lesion classification from dermoscopic images using deep learning techniques", in Proc. of 13th IASTED International Conference on Biomedical Engineering (BioMed), 2017, pp. 49-54. DOI: 10.2316/P.2017.852-053

[18] J. Shihadeh, A. Ansari, and T. Ozunfunmi, "Deep learning based image classification for remote medical diagnosis", in Proc. of 2018 IEEE Global Humanitarian Technology Conference (GHTC), 2018, pp. 1-8. DOI: 10.1109/GHTC.2018.8601558

[19] M. H. Jafari, N. Karimi, E. Nasr-Esfahani, S. Samavi, S. M. R Soroushmehr, K. Ward, and K. Najarian, "Skin lesion segmentation in clinical images using deep learning", in Proc. of 23rd International Conference on Pattern Recognition (ICPR), 2016, pp. 337-342. DOI: 10.1109/ICPR.2016.7899656.

[20] P. Sabouri and H. GholamHosseini, "Lesion border detection using deep learning", in Proc. of 2016 IEEE Congress on Evolutionary Computation (CEC), 2016, pp. 1416-1421. DOI: 10.1109/CEC.2016.7743955.

[21] V. Singh and I. Nwogu, "Analyzing skin lesions in dermoscopy images using convolutional neural networks", in Proc. of 2018 IEEE International Conference on Systems, Man, and Cybernetics (SMC), 2018, pp. 4035-4040. DOI: 10.1109/SMC.2018.00684.

[22] L. Yu, H. Chen, Q. Dou, J. Qin, and P.-A. Heng, "Automated melanoma recognition in dermoscopy images via very deep residual networks", IEEE Transactions on Medical Imaging, vol. 36, no. 4, pp. 994-1004, 2017. DOI: 10.1109/TMI.2016.2642839.

[23] N. C. F. Codella, Q.-B. Nguyen, S. Pankanti, D. A. Gutman, B. Helba, A. C. Halpern, and J. R. Smith, "Deep learning ensembles for melanoma recognition in dermoscopy images", IBM Journal of Research and Development, vol. 61, nos. 4/5, pp. 5:1-5:15, 2017. DOI: 10.1147/JRD.2017.2708299.

[24] C. Li, J. Guo, F. Porikli, and Y. Pang, "LightenNet: A convolutional Neural Network for weakly illuminated image enhancement", Pattern Recognition Letters, vol. 104, pp. 15-22, 2018. DOI: 10.1016/J.PATREC.2018.01.010.

[25] J. Glaister, R. Amelard, A. Wong, and D. A. Clausi, "MSIM: "Multistage illumination modeling of dermatological photographs for illumination-corrected skin lesion analysis", IEEE Transactions on Biomedical Engineering, vol. 60, no. 7, pp. 1873-1883, 2013. DOI: 10.1109/TBME.2013.2244596

[26] E. Zagrouba and W. Barhoumi, "An accelerated system for melanoma diagnosis based on subset feature selection", Journal of Computing and Information Technology, vol. 13, no. 1, pp. 69-82, 2005. DOI: 
10.2498/CIT.2005.01.06.

[27] E. Ayan and H. M. Ünver, "Data augmentation importance for classification of skin lesions via deep learning", in Proc. of 2018 Electric Electronics, Computer Science, Biomedical Engineerings' Meeting (EBBT), 2018, pp. 1-4. DOI: 10.1109/EBBT.2018.8391469.

[28] E. Nasr-Esfahani, S. Samavi, N. Karimi, S. M. R. Soroushmehr, M. H. Jafari, K. Ward, and K. Najarian, "Melanoma detection by analysis of clinical images using convolutional neural network", in Proc. of 2016 38th Annual International Conference of the IEEE Engineering in Medicine and Biology Society (EMBC), 2016, pp. 1373-1376. DOI 10.1109/EMBC.2016.7590963.

[29] R. S. Soumya, S. Neethu, T. S. Niju, A. Renjini, and R. P. Aneesh, "Advanced earlier melanoma detection algorithm using colour correlogram", in Proc. of 2016 International Conference on Communication Systems and Networks (ComNet), 2016, pp. 190-194. DOI: 10.1109/CSN.2016.7824012.

[30] A. Krizhevsky, I. Sutskever, and G. E. Hinton, "ImageNet classification with deep convolutional neural networks", in Proc. of the 25th International Conference on Neural Information Processing Systems, 2012, pp. 1097-1105, vol. 1. DOI: 10.1145/3065386.

[31] Y. Guo, A. S. Ashour, L. Si, and D. P. Mandalaywala, "Multiple convolutional neural network for skin dermoscopic image classification", in Proc. of 2018 IEEE International Symposium on Signal Processing and Information Technology (ISSPIT), 2018, pp. 365-369. DOI: 10.1109/ISSPIT.2018.8642669.

[32] K. M. Hosny, M. A. Kassem, and M. M. Foaud, "Classification of skin lesions using transfer learning and augmentation with Alex-net", PLoS ONE, vol. 14, no. 5, 2019. DOI: 10.1371/JOURNAL.PONE.0217293.

[33] Y. Liu, Y. Chen, P. Lasang, and Q. Sun, "Covariance attention for semantic segmentation", IEEE Transactions on Pattern Analysis and Machine Intelligence, 2020. DOI: 10.1109/TPAMI.2020.3026069.

[34] "Covariance", MATLAB. [Online]. Available: https://www.mathworks.com/help/matlab/ref/cov.html

This article is an open access article distributed under the terms and conditions of the Creative Commons Attribution 4.0 (CC BY 4.0) license (http://creativecommons.org/licenses/by/4.0/). 